\title{
Naturally processed and presented epitopes of the islet cell autoantigen IA-2 eluted from HLA-DR4
}

\author{
Mark Peakman, ${ }^{1}$ Elizabeth J. Stevens, ${ }^{1}$ Tobias Lohmann, ${ }^{2}$ Parth Narendran, ${ }^{3}$ \\ James Dromey, ${ }^{4}$ Angela Alexander, ${ }^{5}$ Andrew J. Tomlinson, ${ }^{6}$ Massimo Trucco, ${ }^{5}$ \\ Joan C. Gorga, ${ }^{5}$ and Roman M. Chicz ${ }^{6}$ \\ ${ }^{1}$ Department of Immunology, and \\ ${ }^{2}$ Department of Medicine, Guy's, King's and St. Thomas' School of Medicine, King's College London, Denmark Hill Campus, \\ London SE5 9PJ, United Kingdom \\ ${ }^{3}$ Department of Internal Medicine III, University of Leipzig, Leipzig 04103, Germany \\ ${ }^{4}$ University Department of Medicine, Bristol Royal Infirmary, Bristol BS2 8HW, United Kingdom \\ ${ }^{5}$ Division of Immunogenetics, Children's Hospital of Pittsburgh, Pittsburgh, Pennsylvania 15213, USA \\ ${ }^{6}$ Zycos Inc., Cambridge, Massachusetts 02138, USA \\ Joan C. Gorga's present address is: Ares Advanced Technology, Randolph, Massachusetts 02368, USA
}

Address correspondence to: Mark Peakman, Department of Immunology, Guy's, King's and St Thomas' School of Medicine, King's College London, Denmark Hill Campus, London SE5 9PJ, United Kingdom. Phone: 44-171-848-5956; Fax: 44-171-848-5953; E-mail: mark.peakman@kcl.ac.uk.

Received for publication July 22, 1999, and accepted in revised form October 5, 1999.

\begin{abstract}
During immune responses, antigen-presenting cells (APCs) process antigens and present peptide epitopes complexed with human leukocyte antigen (HLA) molecules. CD4 cells recognize these naturally processed and presented epitopes (NPPEs) bound to HLA class II molecules. Epitope identification is important for developing diagnostic and therapeutic tools for immune-mediated diseases and providing insight into their etiology, but current approaches overlook effects of natural processing on epitope selection. We have developed a technique to identify NPPEs using mass spectrometry (MS) after antigen is targeted onto APCs using a lectin-based antigen delivery system (ADS). We applied the technique to identify NPPEs of the intracellular domain of the type 1 diabetes mellitus-associated (type 1 DMassociated) autoantigen insulinoma-associated-2 (IA-2ic), presented by HLA-DR4 (0401). IA-2ic-derived NPPEs eluted from HLA-DR 4 constitute 6 sets of peptides nested around distinct core regions. Synthetic peptides based on these regions bind HLA-DR4 and elicit primary T-cell proliferation frequently in HLA-DR4-positive type 1 DM patients, but rarely in non-HLA-DR4 patients, and in none of the HLA-DR4 nondiabetic controls we tested. This flexible, direct approach identifies an HLA allele-specific map of NPPEs for any antigen, presented by any HLA class II molecule. This method should enable a greater understanding of epitope selection and lead to the generation of sensitive and specific reagents for detecting autoreactive $\mathrm{T}$ cells.
\end{abstract}

J. Clin. Invest. 104:1449-1457 (1999).

\section{Introduction}

There is a major interest in defining peptide epitopes recognized by CD4 $\mathrm{T}$ cells involved in immune responses (1), especially in diseases strongly associated with allelic forms of genes in the human leukocyte antigen (HLA) class II region, including certain types of infection $(2,3)$, cancer $(4,5)$, and autoimmune disease (6). In these disorders identification of epitopes recognized by $\mathrm{CD} 4 \mathrm{~T}$ cells is important for understanding mechanisms of disease development (molecular mimicry, for example) (7), for enhancing diagnosis and prediction, and also for the future development of peptide-based therapies and vaccines $(8,9)$. Peptide epitopes derived from exogenous antigens (Ag's) are presented to CD4 T cells after a sequence of events termed natural processing and presentation (1). Native Ag is internalized and then cleaved by a combination of enzymatic and chemical events, giving rise to peptides that bind HLA class II molecules for export to the antigen-presenting cell (APC) surface (10). Natural processing gives rise to large nested sets of pep- tides that are bound to HLA class II molecules through the same core motif, but they are variably extended and truncated at the $\mathrm{NH}_{2}$ - and $\mathrm{COOH}$-termini (11). The $\mathrm{NH}_{2}-$ and $\mathrm{COOH}$-terminus peptide flanking residues (PFRs) can have potent effects on HLA binding and activation of CD4 T-cell clones (12-14). For example, Carson et al. have shown that CD4 T-cell clones specific for the I-Ak immunodominant epitope of hen egg lysozyme (HEL) 52-61 are often entirely dependent upon, and specific for, $\mathrm{COOH}$-terminal PFRs that lie outside the core MHCbinding region (14). In the same study, HEL peptides containing PFRs were considerably more immunogenic and mediated a greater recall response to the HEL protein than truncated peptides that were still bound to MHC.

At present, the most widely used approach to epitope identification is the construction of sets of overlapping synthetic peptides, spanning the Ag of interest. This approach is unable to identify naturally processed and presented epitopes (NPPEs), however, and is therefore unable to direct studies toward defining those PFRs that 
significantly enhance epitope recognition by CD4 T cells. For these reasons, we elected to develop a system for the direct identification of peptides naturally processed from specific Ag's and presented by HLA class II molecules as NPPEs recognized by CD4 T cells. We have applied the new technology to the prototypic organ-specific autoimmune disease, type 1 diabetes mellitus (DM), in which immune responses to numerous islet cell autoantigens occur on a distinctive genetic background, notably the possession of HLA-DRB1*0401, $D Q A 1 * 0301 / D Q B 1 * 0302$ [DQ3.2] genotypes $(15,16)$. In the present study we have focused on the islet cell autoantigen insulinoma-associated-2 (IA-2), one of a family of protein tyrosine phosphatases (PTPs) (17). Autoantibodies against these PTPs are directed toward the intracellular domain (IA-2ic), almost without exception (18), and are associated with rapid progression to diabetes in high-risk subjects (19). In the present study we identify NPPEs of IA-2ic bound to HLA-DR4 (0401) and demonstrate that synthetic peptides based on these are sensitive and specific reagents in the detection of autoreactive T cells in HLA-DR4 type 1 DM patients.

\section{Methods}

Subjects. Fresh heparinized and clotted blood samples were obtained from 21 Caucasian type $1 \mathrm{DM}$ patients (median age 20 years, range $6-42$ years) recruited a median of 8 weeks after diagnosis (range 1-28 weeks). All had acute onset of symptoms, ketosis, and required insulin from diagnosis. Samples were also collected from healthy Caucasian nondiabetic control subjects without history of first-degree relatives with type 1 DM (median age 24 years, range 16-36 years) and from 13 patients with rheumatoid arthritis (median age 62 years, range 35-74 years). PBMCs were isolated on density gradients (Lymphoprep; Nycomed-Amersham, Buckingham, United Kingdom) and washed in HBSS (Life Technologies, United Kingdom) twice before use. Subjects were HLA genotyped as described (20), and IA-2ic autoantibodies were detected by immunoprecipitation of in vitro transcribed and translated recombinant protein as described (21).

Antigens. Biotinylated IA-2ic (b-IA-2ic) was generated as described (22); briefly, E coli strain JM109 cells were transformed with cDNA for IA-2ic representing amino acids 603-979 (provided by Michael Christie) cloned in the expression vector Pinpoint (Promega UK Ltd., Southampton, United Kingdom). This vector produces a fusion protein of IA-2ic coupled at the $\mathrm{NH}_{2}$ terminus to a biotinylated 130 amino acid leader sequence comprising the $1.3 \mathrm{~S}$ subunit of Propionobacterium shermanii transcarboxylase. This enables purification on avidinagarose and provides a biotinylated form of the $\mathrm{Ag}$ for direct use in the antigen delivery system (ADS). After elution from the avidin-agarose column (Promega), IA-2ic was separated from excess biotin using a Sephadex G25 column (Pharmacia Biotech AB, Uppsala, Sweden) and concentrated using an Amicon B15 concentrator (Amicon Ltd., Stonehouse, Gloucestershire, United Kingdom). Biotinylated IA-2ic was typically more than 95\% pure by SDS-PAGE and Coomassie staining. Levels of endotoxin ranged between 30 and $50 \mathrm{EU}$ per $50 \mu \mathrm{g}$ recombinant IA-2ic. Tetanus toxoid (TT; Connaught
Laboratories, Pittsburgh, Pennsylvania, USA) was biotinylated by incubation at room temperature for 2 hours with biotin (long-arm) $N$-hydroxysuccinimide (Vector Laboratories Ltd., Peterborough, United Kingdom) at $10 \%$ of the mass of the Ag, according to the manufacturer's instructions. The reaction was halted by addition of glycine, and unreacted biotin was removed by gel filtration using a Sephadex G25 column.

Antigen delivery to Epstein-Barr virus-transformed B cells. Recombinant IA-2ic was delivered to the surface of APCs using an ADS similar to that previously described (22). In the present work the ADS comprised biotinylated pokeweed mitogen (b-PWM) that binds preferentially to carbohydrate moieties on surface receptors with immunoglobulin-like domains, such as the B-cell receptor complex on to Epstein-Barr virus-transformed (EBVtransformed) B cells (23). Avidin was then used as a bridge between cell surface-bound b-PWM and b-IA-2ic (Figure 1a). As in our previous studies (22), the presence of the biotinylated Ag on the APC surface, delivered through the ADS, was demonstrated by flow cytometry using a FACScan (Becton Dickinson UK Ltd., Oxford, United Kingdom). To establish optimal pulsing conditions, cells were pulsed with varying concentrations of bPWM, avidin-D, and biotinylated TT (b-TT), and then incubated in RPMI $/ 10 \% \mathrm{FCS}$ at $37^{\circ} \mathrm{C}, 5 \%$ carbon dioxide $\left(\mathrm{CO}_{2}\right)$ for $0,1,3$, or 6 hours, after which aliquots were immediately placed on ice. Cells were stained for $30 \mathrm{~min}$ utes with polyclonal rabbit anti-TT antiserum (Biogenesis Ltd., Poole, United Kingdom), washed in HBSS, and then stained with FITC goat anti-rabbit IgG (Becton Dickinson). After washing and resuspending in precooled PBS, binding was examined by flow cytometry, with a minimum of 10,000 events acquired for each sample.

Priess EBV B cells, homozygous for the type 1 DM-permissive DRB1*0401, [DR4/DRw53], $D Q A 1 * 0301 / D Q B 1 * 0302$ [DQ8] genotype were harvested as APCs, washed with HBSS, counted, and resuspended in HBSS at $10^{8} / \mathrm{mL}$ on ice. Cells were then pulsed sequentially on ice for 30 minutes with each component of the ADS at optimal concentrations, comprising b-PWM $(1 \mu \mathrm{g} / \mathrm{mL}$; Sigma Chemical Co., Poole, United Kingdom), avidin-D (2 mg/mL; Vector Laboratories), and recombinant b-IA-2ic (20 $\mu \mathrm{g} / \mathrm{mL}$ ), with 2 washing steps with excess cold HBSS between each pulse. Pulsed Priess cells were then incubated in RPMI 1640/10\% FCS (Life Technologies) at $10^{6} / \mathrm{mL}$ for 1 or 6 hours at $37^{\circ} \mathrm{C}, 5 \%$ carbon dioxide $\left(\mathrm{CO}_{2}\right)$. Under identical conditions, an equivalent number of Priess cells were pulsed with b-PWM/avidin-D but not b-IA-2ic (control cells). At the end of the incubation period, pulsed Priess cells were washed in HBSS and stored at $-80^{\circ} \mathrm{C}$.

To examine whether the lectin-based ADS delivers relevant peptide epitopes bound to HLA-DR4 on the Priess cell surface, TT-specific CD4 T-cell lines were expanded from the peripheral blood of a healthy control subject (homozygous for HLA-DR4 [0401])using 2-3 successive rounds of TT $(10 \mu \mathrm{g} / \mathrm{mL}, 5$ days $)$ and IL-2 $(30 \mathrm{U} / \mathrm{mL}, 3$ days) stimulation as described $(22,24)$. Priess cells were loaded with b-TT using the ADS as described above. Proliferation of TT-specific T-cell line cells to Priess cells 
pulsed with the ADS and b-TT was measured by ${ }^{3} \mathrm{H}-$ thymidine incorporation as described (22) and compared with proliferation to soluble TT presented conventionally by autologous irradiated PBMC.

Purification of HLA-DR4 and peptide elution and analysis by mass spectrometry. HLA-DR4 was purified from Priess cell pellets pulsed with the ADS as described previously (25). Briefly, cell pellets were homogenized in hypotonic buffer and a crude membrane fraction solubilized in 4\% NP-40 (Sigma Chemical Co.). The detergent-soluble fraction was passed over a series of immunoaffinity columns made with $m A$ bs specific for HLA class I proteins (W6/32), HLADR (L243) coupled to protein A-Sepharose, and HLADQ8 (IVD12) coupled to Affigel-10 (Bio-Rad Laboratories, Ltd., Hemel Hempstead, United Kingdom). The immunoaffinity columns were eluted with $50 \mathrm{mM}$ glycine, pH 11.5, $0.1 \%$ sodium deoxycholate, and were immediately neutralized and dialyzed against $10 \mathrm{mM}$ Tris, $\mathrm{pH}$ 8.0, and $0.1 \%$ sodium deoxycholate. HLA-DR4 was more than $98 \%$ pure as assessed by SDS-PAGE. Immediately before acid extraction of bound peptides, a $0.5-\mathrm{mg}$ aliquot of HLA protein was passed through an HPLC size-exclusion column (ProGel TSK G2000 SW; $7.5 \times 300$ mm; Waters Corp., Milford, Massachusetts, USA) equilibrated with 10 $\mathrm{mM}$ Tris, $\mathrm{pH} 7.5$, to remove molecules not specifically bound to the HLA protein. Fractions containing the HLADR4 proteins were concentrated to $100 \mu \mathrm{L}$ by ultrafiltration (Centricon 10; Amicon Ltd.).

Naturally processed peptide repertoires were acid eluted by incubation for 15 minutes at $70^{\circ} \mathrm{C}$ with $800 \mu \mathrm{L}$ $10 \%$ acetic acid and isolated from the remaining HLA protein by ultrafiltration through the Centricon 10 . Acid-extracted peptides were vacuum concentrated to approximately $20-30 \mu \mathrm{L}$ and separated by reverse phase (RP)-HPLC, using previously reported gradient conditions (26) and a microbore $\mathrm{C}_{18}$ column $(1.0 \times 250 \mathrm{~mm}$; Vydac, Hesperia, California, USA) at $50 \mu \mathrm{L} /$ minute. The column effluent was split such that $2 \%$ was immediately loaded onto a sample plate along with $0.4 \mu \mathrm{L}$ of matrix ( $\alpha$-cyano-4-hydroxycinnamic acid, $10 \mathrm{mg} / \mathrm{mL}$ in $50 \%$ acetonitrile $/ 0.1 \%$ trifluoroacetic acid) and allowed to air dry. Mass spectra were collected at optimum laser intensities by averaging the ion signals from 128 individual scans in both linear and reflector modes using a single-stage extended-length reflector time-of-flight mass spectrometer (Voyager Elite XL; PerSeptive Biosystems, Framingham, Massachusetts, USA). Time to mass conversion was performed by external calibration using synthetic peptides. Mass accuracy varied from $0.03 \%$ to better than $0.01 \%$ in linear and reflector modes, respectively. An automated microcapillary liquid chromatography-mass spectrometry (LC-MS; Finnegan Inc., San Jose, California, USA) approach with data-dependent collision-activated dissociation (CAD) for sequencing low levels of NPPEs ( $1 \mathrm{fmol}$ ) was developed to directly sequence targeted peptide masses determined by matrix-assisted laser desorption ionization time-of-flight MS (MALDI-TOFMS), as described above. Peptide fractions were further separated using a second dimension microcapillary reverse-phase support (internal diameter $75 \mu \mathrm{m}$ packed with $7 \mu \mathrm{m} 200$ Å Brownlee C18 material). CAD experiments were triggered in a data-dependent mode, using ions that are more abundant than an operator-set threshold. The electrospray voltage was $3.5 \mathrm{kV}$.

Peptide synthesis, HLA-DR4 binding assay, and T-cell proliferation assays. Identified peptide fragments of naturally processed IA-2ic were synthesized using an Applied Biosystems SYNERGY peptide synthesizer (Perkin Elmer Ltd., Beaconsfield, United Kingdom) and amino acid sequence, and purity of greater than $95 \%$ was confirmed by RP-HPLC and MALDI-TOF MS. Binding of IA-2ic peptides to affinity-purified, soluble HLA-DR4 (0401) was measured by direct competition with biotinylated invariant chain (Ii) peptide PKPPKPVSKMPMATPLLMQA as described previously (27). Briefly, $0.1 \mu \mathrm{g}$ immunoaffinity-purified HLA-DR4 protein was incubated with $2.5 \mu \mathrm{M}$ biotinylated Ii peptide and the test competing peptide or nonbiotinylated Ii peptide at a range of concentrations $(0.1-100 \mu \mathrm{M})$ in $20 \mathrm{mM} \mathrm{2-[N-}$ morpholino]ethanesulfonic acid, $140 \mathrm{mM}$ sodium chloride $(\mathrm{NaCl}), 0.05 \%$ sodium azide $\left(\mathrm{NaN}_{3}\right), \mathrm{pH}$, overnight at room temperature. Incubations were transferred to microtiter plate wells that had been precoated with $100 \mu \mathrm{L}$ anti-HLA-DR (clone L243) at $10 \mu \mathrm{g} / \mathrm{mL}$ in PBS, blocked with 3\% nonfat dried milk and 3\% BSA for 30 minutes each, and washed 5 times with $10 \mathrm{mM}$ Tris, $140 \mathrm{mM} \mathrm{NaCl}, 0.05 \%$ Tween 20, pH 8 (TBST). The peptide/protein mixtures were incubated at room temperature for 1 hour and washed 5 times in TBST. Streptavidin-alkaline phosphatase (Boehringer Mannheim, Lewes, East Sussex, United Kingdom) was added at a final concentration of $1 \mathrm{U} / \mathrm{mL}$ in TBST $/ 1 \%$ BSA and incubated for 1 hour before development with $p$-nitrophenylphosphate diluted to $1 \mathrm{mg} / \mathrm{mL}$ in $1: 4$ diethanolamine/water. Absorbance was read after 20-40 minutes at $405 \mathrm{~nm}$.

For T-cell proliferation assays, $10^{5}$ peripheral blood mononuclear cells were cultured in 12 replicates in 96well U-bottom plates with peptides $(10 \mu \mathrm{g} / \mathrm{mL})$ for 5 days in RPMI 1640/10\% human AB serum, followed by pulsing for 18 hours with $0.5 \mu \mathrm{Ci}^{3} \mathrm{H}$-thymidine per well and harvesting onto filters for radioactivity counting. Control wells consisted of 12 replicates cultured in the absence of peptide on each test plate. Control wells from both test plates were used to calculate the mean cpm +2 $\mathrm{SD}$, above which test wells were considered positive. Tcell proliferation was considered significant only if the stimulation index (ratio of mean specific test proliferation to mean of wells containing medium alone) was greater than 2 and the frequency of wells positive was significant compared with control wells (for example, $5 / 12$ test wells positive is significantly higher than $0 / 24$ control wells by $\chi^{2}$ analysis; $\left.P<0.05\right)$. Results were not different when 12 or 24 replicate background wells were used in the calculations. These criteria are similar to those applied in similar studies of peripheral blood responses to peptide autoantigens $(28,29)$.

\section{Results}

Lectin-based ADS loads biotinylated antigen onto Priess cells, leading to rapid internalization and presentation of relevant epitopes recognized by $C D 4 T$ cells. We targeted biotinylated Ag's onto the surface of the Priess EBV-transformed B cell line using an ADS (Figure 1a). Flow cytometry was used to examine 
the time course of Ag delivery. Surface b-TT was internalized rapidly and had disappeared from the Priess cell surface after 6 hours of incubation at $37^{\circ} \mathrm{C}$ (Figure $1 \mathrm{~b}$ ).

To establish whether the ADS leads to delivery of physiologically relevant NPPEs bound to class II HLA molecules on the APC surface, Priess cells loaded with b-TT through the ADS were examined for their ability to induce proliferation in TT-specific CD4 T-cell lines. A representative T-cell line, NG2, showed similar high levels of ${ }^{3} \mathrm{H}$-thymidine incorporation when cocultured with APCs (Priess EBV cells) pulsed with b-TT using the ADS (mean $\Delta \mathrm{cpm}=7750$ after 3 days), as when APCs (autologous irradiated PBMCs) were incubated with TT in simple solution (mean $\Delta \mathrm{cpm}=8427$; mean background counts $2528 \mathrm{cpm})$. As with our previous experience of targeting Ag's onto APCs using an ADS (22), these experiments show that proteins delivered through the ADS are internalized rapidly, directed into the class II Ag processing pathway, and that relevant epitopes are presented.

Analysis of peptides eluted from HLA-DR4 purified from IA2ic-pulsed Priess cells. We then set out to elute and identify NPPEs derived from IA-2ic after loading of Priess cells using the ADS. The b-PWM/avidin ADS was used to pulse $5-10 \times 10^{9} \mathrm{EBV}$ B cells with b-IA-2ic, followed by incubation for 1 or 6 hours at $37^{\circ} \mathrm{C}$ to allow internalization, processing, and presentation. The same number of cells were pulsed with b-PWM/avidin alone (control preparation). HLA-DR4 (0401) was purified from each cell pellet, bound peptides were eluted and separated by RP-HPLC, and each of 100 fractions were analyzed by MALDI-TOF-MS. RP-HPLC analysis was highly reproducible, with no discernible differences between chromatographic traces from the IA-2ic-pulsed and control HLA-DR4 preparations (Figure 2). The mass spectra for the intact HLA-DR4 (0401) peptide repertoire isolated from Priess cells pulsed with IA-2ic were compared with the spectra for the peptide repertoire isolated from control Priess cells to identify novel $\mathrm{m} / \mathrm{z}$ values corresponding to peptides derived from IA-2ic (Figure 3 ).

Of the approximately $6000 \mathrm{~m} / \mathrm{z}$ values corresponding to the NPPEs isolated from the IA-2ic-pulsed and control-

\section{Figure 1}

ADS delivers antigen to the EBV B-cell surface at high concentration. (a) The b-PWM has preferential affinity for disulfide-bonded Ig-like molecules such as surface Ig on the EBV B cell. The b-PWM is then bound to avidin, which can bind up to 3 molecules of biotinylated antigen. (b) The PWM-based ADS is completely internalized in 6 hours. Priess EBV B cells were incubated sequentially on ice with each component of the ADS (bPWM, avidin-D, biotinylated antigen [TT]) and then incubated in culture medium at $37^{\circ} \mathrm{C}$ for $0,1,3$, or 6 hours and removed for analysis of surface $\Pi T$ by flow cytometry, using rabbit anti-TT antiserum and FITC goat anti-rabbit Ig. Compared with background (solid line), surface expression was high at 0 hours (long dashes) but had diminished by 1 (dashes broken by dots) and 3 hours (short dashes). ADS-delivered antigen was completely internalized by 6 hours (dotted line). pulsed cells, 85 masses were identified as being unique to the IA-2ic-pulsed NPPE preparation. Upon further mass analysis using higher resolution and more stringent mass accuracy, the unique mass values were mass mapped against the linear sequence of IA-2ic, revealing 24 mass matches. The mass identification was highly reproducible, with the same 24 masses being identified in two separate EBV B-cell preparations and subsequent NPPE isolation. The same masses were seen when EBV B cells were allowed to internalize, process, and present Ag for 1 hour and 6 hours, although better peptide loading was seen at 1 hour. The 24 masses identified correspond to 35 IA-2ic sequences shown in Table 1 . All 35 sequences are members of 6 nested sets of peptides. Nested sets are groups of peptides based around the same core region, but variably truncated or extended at the $\mathrm{NH}_{2}$-and/or COOH-termini, and are characteristic of processing through the class II endocytic pathway (11). All 6 core regions contain amino acid binding preferences for HLA-DR4 (0401)(30). HPLC coupled with electrospray MS (LC-MS) was used to verify the amino acid sequences of several candidate NPPEs from the IA-2ic pulsed cells. Partial fragmentation patterns corresponding to ESGCTVIVMLTPLVEDG, YLKNVQTWQETRTL, and YLKNVQTQETRTLTQ were obtained (Table 1).

Sequence analyses of the 61 novel masses not mapped to IA-2ic revealed nested sets of NPPEs derived from hsp 70, G-M2 activator protein, HLA class I, and several other self proteins. Presumably, upregulated processing of self proteins occurred as a result of pulsing with recombinant IA-2ic or bacterial contaminants (e.g., endotoxin) in the preparation.

HLA-DR4 binding and spontaneous T-cell proliferation to synthetic peptides based on IA-2ic NPPEs. Synthetic peptides based on the 6 core regions of IA-2ic (Table 1) were used to examine binding to HLA-DR4 and spontaneous peripheral blood T-cell responses in type $1 \mathrm{DM}$ patients with and without HLA-DR4 and in HLA-DR4 healthy control subjects. Peptides were synthesized so that they included putative core HLA-DR4 binding residues, based on published amino acid preferences at P1 and P6 $(26,31)$, and were then extended at the $\mathrm{NH}_{2}$-termini to provide

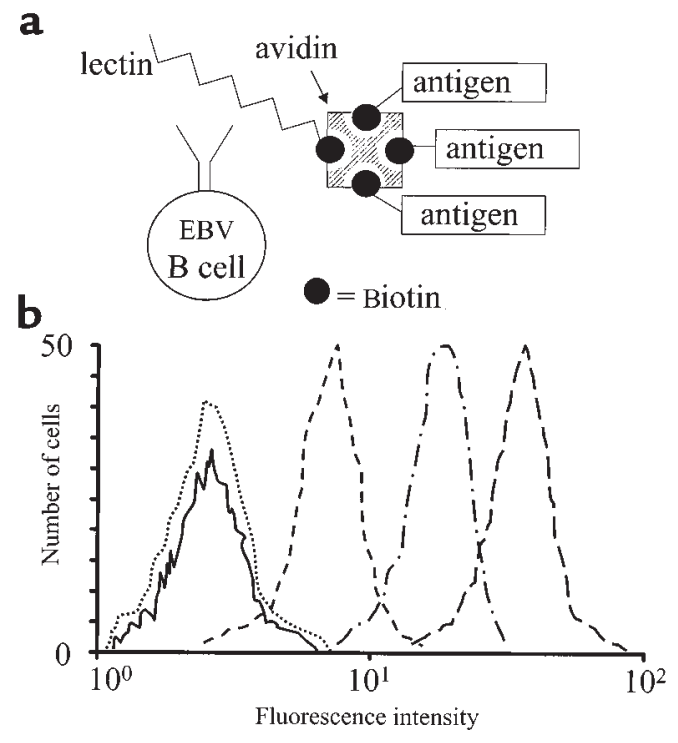


Table 1

Experimentally observed and calculated masses of IA-2-derived peptides eluted from HLA-DR4 (0401)

\begin{tabular}{|c|c|c|c|c|}
\hline Observed $\mathrm{m} / \mathrm{z}$ & Calculated $\mathrm{m} / \mathrm{z}$ & Residues & $\begin{array}{l}\text { Corresponding } \\
\text { IA-2ic sequence }\end{array}$ & $\begin{array}{l}\text { Synthetic peptide used } \\
\text { in primary T-cell assay }\end{array}$ \\
\hline 1469.31 & 1468.65 & $657-671$ & VSSQFSDAAQASPSS & $654-674$ \\
\hline 1469.31 & 1468.65 & $656-670$ & SVSSQFSDAAQASPS & VSSVSSQFSDAAQASPSSHSS \\
\hline 1469.31 & 1468.65 & $655-669$ & SSVSSQF SDAAQASP & \\
\hline 1866.76 & 1866.80 & $656-674$ & SVSSQF SDAAQASP SSHSS & \\
\hline 2397.16 & 2397.08 & $652-675$ & SRVSSVSSQFSDAAQASPSSHSST & \\
\hline 2441.48 & 2441.02 & $656-679$ & SVSSQFSDAAQASPSSHSSTPSWC & \\
\hline 2483.29 & 2483.03 & $657-680$ & VSSQF SDAAQASPSSHSSTPSWCE & \\
\hline 1367.44 & 1367.57 & $718-730$ & AYQAEPNTCATAQ & $709-732$ \\
\hline 1640.86 & 1640.68 & $716-731$ & LCAYQAEPNTCATAQG & LAKEWQALCAYQAEPNTCATAQG \\
\hline 1935.80 & 1935.91 & $709-725$ & LAKEWQALCAYQAEPNT & $\mathrm{E}$ \\
\hline 1965.83 & 1965.88 & $718-736$ & AYQAEPNTCATAQGEGNIK & \\
\hline 1968.75 & 1968.84 & $713-730$ & WQALCAYQAEPNTCATAQ & \\
\hline 1367.44 & 1367.37 & $754-765$ & KVESSPSRSDYI & \\
\hline 1367.44 & 1367.67 & $753-764$ & LKVESSPSRSDY & $753-771$ \\
\hline 1880.98 & 1880.96 & $752-768$ & KLKVESSPSRSDYINAS & LKVESSPSRSDYINASP I I \\
\hline 2441.48 & 2441.19 & $754-775$ & KVESSPSRSDY INASP I IEHDP & \\
\hline 1489.64 & 1489.75 & $802-815$ & GCTVIVMLTPLVED & $797-817$ \\
\hline 1489.64 & 1489.75 & $803-816$ & CTVIVMLTPLVEDG & MVWESGCTVIVMLTPLVEDGV \\
\hline 1762.88 & 1762.85 & $800-816$ & ESGCTVIVMLTPLVEDG & \\
\hline 1779.75 & 1780.19 & $797-812$ & MVWESGCTVIVMLTPL & \\
\hline 1861.16 & 1860.97 & $800-817$ & ESGCTVIVMLTPLVEDGV & \\
\hline 1883.44 & 1882.88 & $795-810$ & WQMVWESGCTVIVMLT & \\
\hline 2144.95 & 2144.97 & $793-810$ & DFWQMVWESGCTVIVMLT & \\
\hline 2341.17 & 2339.15 & $794-813$ & FWQMVWESGCTVIVMLTPLV & \\
\hline 1508.65 & 1508.74 & $861-872$ & TQETRTLTQFHF & $854-872$ \\
\hline 1593.34 & 1593.85 & $855-867$ & YLKNVQTQETRTL & FYLKNVQTQETRTLTQFHF \\
\hline 1735.78 & 1735.87 & $859-872$ & VQTQETRTLTQFHF & \\
\hline 1806.79 & 1806.96 & $856-870$ & LKNVQTQETRTLTQF & \\
\hline 1822.41 & 1822.96 & $855-869$ & YLKNVQTQETRTLTQ & \\
\hline 1831.87 & 1830.94 & $857-871$ & KNVQTQETRTLTQFH & \\
\hline 2341.17 & 2341.19 & $853-871$ & SFYLKNVQTQETRTLTQFH & \\
\hline 1469.31 & 1469.67 & $957-969$ & DQFEFALTAVAEE & $955-975$ \\
\hline 1866.76 & 1866.90 & $957-973$ & DQFEFALTAVAEEVNAI & SKDQFEFALTAVAEEVNAILK \\
\hline 1935.49 & 1936.04 & $959-976$ & FEFALTAVAEEVNAILKA & \\
\hline 1968.75 & 1968.95 & $955-972$ & SKDQFEFALTAVAEEVNA & \\
\hline
\end{tabular}

optimal hydrogen-binding contacts between the peptide backbone and HLA-DR4. In addition, $\mathrm{NH}_{2}$-and $\mathrm{COOH}$ termini extensions were included to provide amino acids to act as PFRs capable of interaction with the TCR.

All 6 IA-2ic peptides bound HLA-DR4 (Figure 4). Binding affinities for test peptides ranged from strong competition with the Ii peptide (IA-2ic peptides 654-674, 709-732, and 797-817), to those peptides that competed weakly (753-771, 854-872, and 955-975).

Of 13 HLA-DR4 type $1 \mathrm{DM}$ patients, 9 had significant T-cell proliferative responses to one of the 6 peptides (Table 2, Figure 5). Only $1 / 8$ non-DR4 type 1 DM patients showed T-cell proliferation to any of the peptides. None of the nondiabetic HLA-DR4 control subjects (all 0401) responded to any of the peptides. The Tcell proliferation seen in 1 type $1 \mathrm{DM}$ patient with HLA-DR 1/3 (case 14) presumably reflects the well-documented promiscuity of peptide presentation by class II HLA molecules (26).

None of the 13 patients with rheumatoid arthritis, 6 of whom were HLA-DR4, showed T-cell responses to any of the IA-2ic peptides. None of the patients or controls responded to a control IA-2 peptide (873-885) that binds HLA-DR4 but does not have a mass match to any of the eluted peptides.

Peptides from $5 / 6$ of the core regions elicited T-cell responses, and all but 2 responder patients proliferated to a single peptide. None of the subjects had spontaneous T-cell proliferative responses to peptide 753-771; however, spontaneous HLA-DR4 (0401)-restricted T-cell responses to this region have been observed by others in high-risk first-degree relatives of type $1 \mathrm{DM}$ patients (28), suggesting that this may be an epitope recognized during the early stages of disease. Eleven of the 13 HLADR4 patients had the DRB ${ }^{*} 0401$ genotype, while two patients (both peptide responders) were 0403/0405 heterozygotes. The $D R B 1 * 0403$ and 0405 genes encode similar DR $\beta$ chains to 0401 , differing only at positions 57 (0405 S for D), 71 (0403 and $0405 \mathrm{R}$ for K), 74 (0403 E for A), and 86 (0403 V for $\mathrm{G}$ ) (31). The amino acid preferences for peptide to these HLA-DR4 subtypes are similar, and all are predicted to bind to the 6 IA-2ic core peptide regions identified.

To further define the T-cell response to the NPPE regions of IA-2ic, we also synthesized IA-2ic peptides that partially overlapped those shown in Table 1 and tested type 1 DM patients $(n=8)$ and controls $(n=8)$ for whom sufficient cells were available for T-cell proliferation. These peptides were $716-732,753-765,800-814$, and 854-870. T-cell responses and HLA-DR4 binding to these peptides were similar to the index peptides shown in Table 1.

Autoantibodies to IA-2ic were found in $10 / 13$ of the 




Figure 2

RP-HPLC analysis of peptides eluted from HLA-DR4 purified from Priess cells pulsed with ADS plus IA-2ic (upper trace) or ADS alone (lower trace). The chromatograms are largely identical.

HLA-DR4 type 1 DM patients and $2 / 8$ of the non-HLA-DR 4 type $1 \mathrm{DM}$ patients. There did not appear to be any relationship between possession of autoantibodies and T-cell reactivity to IA-2ic, because 3 patients without autoantibodies showed significant T-cell responses. Several studies have now documented the lack of direct relationship between islet autoantibodies and the detection of T-cell reactivity to the same antigen $(29,32,33)$. Patients and control subjects did not differ in T-cell proliferation to TT or PHA. In subjects with sufficient cells available (patients 1-3, 12, and controls 22, 24, and 29; see Table 1), T-cell assays with TT peptides known to elicit T-cell responses (p459 VRDIIDDFTNESSQKT and tt 590-604 TKIYSYFPSVISKVN) were performed. All gave positive responses to one or both peptides, with stimulation indices ranging from $2-12$, with no observed difference between patients and controls.

\section{Discussion}

In the present study we demonstrate that naturally processed HLA class II allele-specific epitopes recognized by $\mathrm{CD} 4 \mathrm{~T}$ cells can be identified after native $\mathrm{Ag}$ is delivered efficiently to EBV-transformed B cells and peptides eluted and analyzed by sensitive and accurate MS. Synthetic peptides based on NPPEs of the islet autoantigen IA-2ic that we eluted from HLA-DR4 prove to be sensitive and specific diagnostic reagents for the detection of autoreactive $T$ cells in patients with type 1 DM and HLA-DR4. The approach benefits from being broadly applicable to any Ag, eluted from any class II MHC molecule, in any disease.

The identification of epitopes of autoantigens, infectious agents, and tumor Ag's recognized by CD4 T cells has been a major research goal in recent years (1). The most widely adopted approach is the synthesis of over-

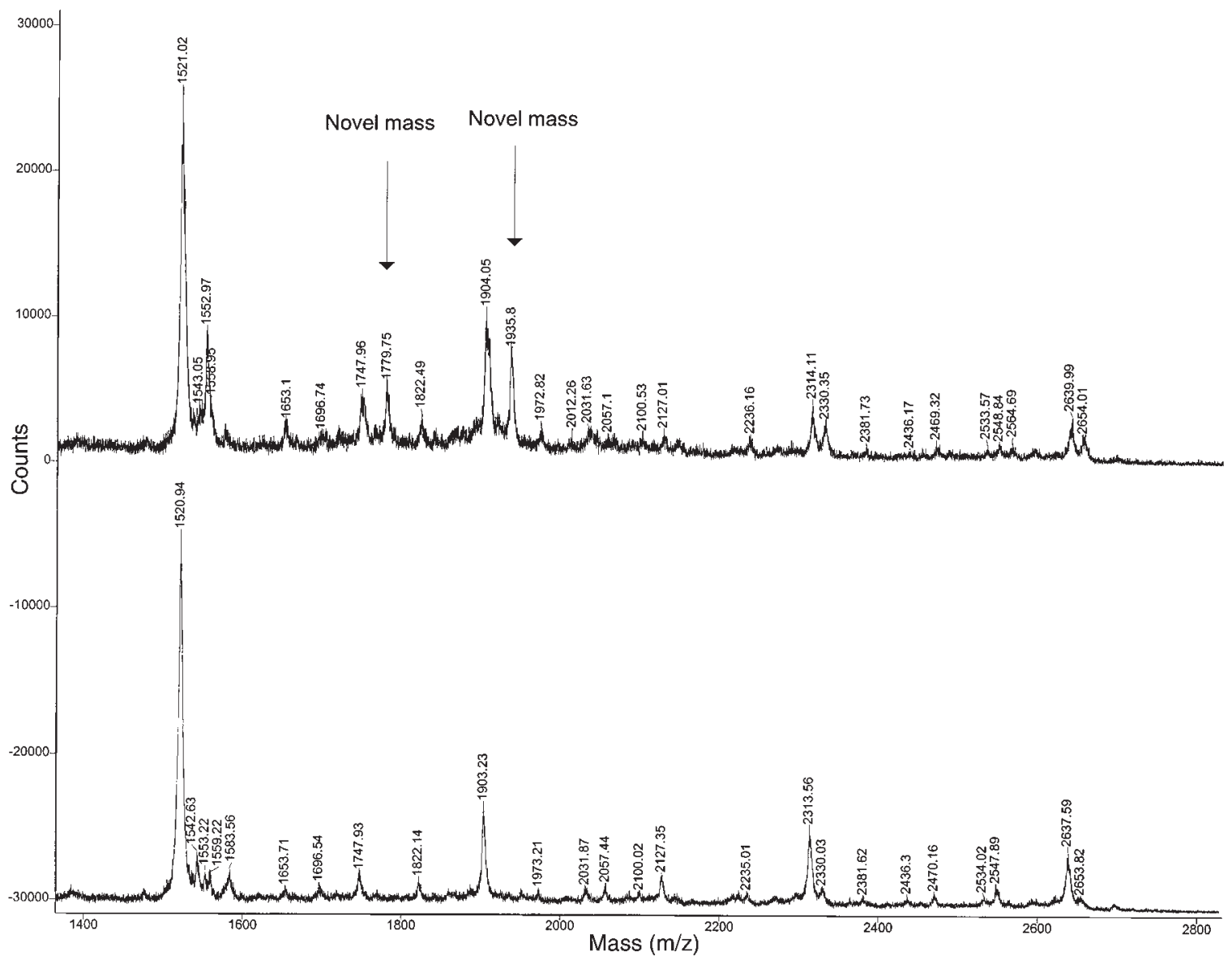

Figure 3

Subtractive approach to identification of IA-2ic-derived, naturally processed and presented peptides by mass spectrometric analysis of representative RP-HPLC fraction of IA-2ic and control-pulsed EBV B cells. Peptide masses in the upper trace have been eluted from HLA-DR4 from Priess EBV $B$ cells pulsed with IA-2ic using the ADS; in the lower trace, B cells were pulsed with the ADS components but no IA-2ic. IA-2ic-derived peptides are marked in the upper trace as novel masses (see Table 1). 


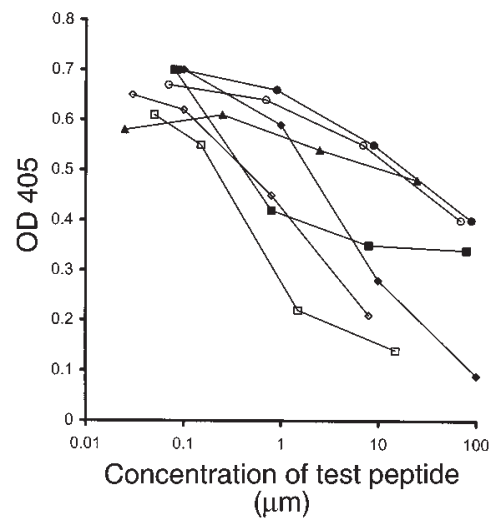

Figure 4

Binding of IA-2ic peptides to HLA-DR4 (0401). Peptides were tested at a range of concentrations for the ability to compete with a fixed concentration $(2.5 \mu \mathrm{M})$ of biotinylated invariant chain (li) peptide. Filled diamonds represent the binding of nonbiotinylated li peptide competing with its biotinylated form. Test peptides of IA-2ic representing residues 654-674 (open squares), 709-732 (open diamonds), and 797-817 (filled squares) are good HLA-DR4 binders, having higher affinity than li. Test peptides of IA-2ic representing residues 753-771 (open circles), 854-872 (filled circles), and 955-975 (filled triangles) bind HLA-DR4, but with lower affinity than li.

lapping peptides spanning the Ag of interest, which are then employed alone, or in pools, to measure binding to HLA class II molecules or T-cell reactivity. There are several problems with these approaches, however. Binding studies are difficult to interpret, because peptides that stimulate CD4 $\mathrm{T}$ cells have widely differing affinities for class II HLA molecules $(28,34)$. Moreover, the relationship between binding affinity and T-cell reactivity is complex, with some poorly binding self peptides capable of potent T-cell stimulation and even induction of autoimmune disease (35). Raising T-cell clones through stimulation with whole Ag for subsequent epitope identification using overlapping peptides is often difficult in human diseases and may give highly selective information (36). On the other hand, when human CD4 T-cell clones have been successfully generated using synthetic peptides, they are frequently unable to recognize the naturally presented native Ag, either as a result of the peptide representing a cryptic epitope, not naturally processed or presented under normal conditions, or the $\mathrm{T}$-cell clone being inadvertently generated against contaminants within the peptide preparation (37-39).

Studies on primary CD4 T-cell proliferation to sets of overlapping peptides have the disadvantage of requiring large volumes of peripheral blood from HLA-matched patients and controls. More importantly, however, the use of sets of arbitrary overlapping peptides for epitope identification does not provide any indication as to whether a particular peptide will be naturally processed and cannot differentiate between false positives and real epitopes naturally presented by APCs. This information can be obtained only by direct elution from class II HLA molecules isolated from APCs. Although it has been possible to identify NPPEs for endogenous self proteins naturally presented by resting APCs $(11,26,27,40)$, there are very few studies that have attempted to identify NPPEs for selected exogenous Ag's. Several of these have focused on HEL, making use of Ag-specific B-cell hybridomas specific for the intact protein to facilitate internalization into Ag processing compartments $(41,42)$. It is difficult to adopt this approach in human disease. For example, although a limited number of EBV-transformed B-cell lines with surface IgG specific for islet autoantigens have been generated, most have been derived from polyendocrine patients whose HLA class II genes are not of relevance to type $1 \mathrm{DM}(43,44)$. An alternative approach has been adopted to study naturally processed and presented epitopes from the Goodpasture autoantigen, in which EBV B cells bearing HLA-DR15 were cultured for 24 hours after pulsing for 1 hour in the presence of recombinant Ag (45). However, insufficient peptide material was obtained in that study for full characterization of putative NPPEs, presumably because of inefficient passive Ag internalization by B cells (11).

To circumvent these problems, in the present study we used a highly efficient ADS to deliver Ag to the APC surface, and we have shown that Ag delivered in this way is rapidly internalized. This is likely to be attributable to effects of PWM on upregulation of Ag processing, because it is a potent B-cell activator. We attribute our success in identifying NPPEs, in some cases obtaining

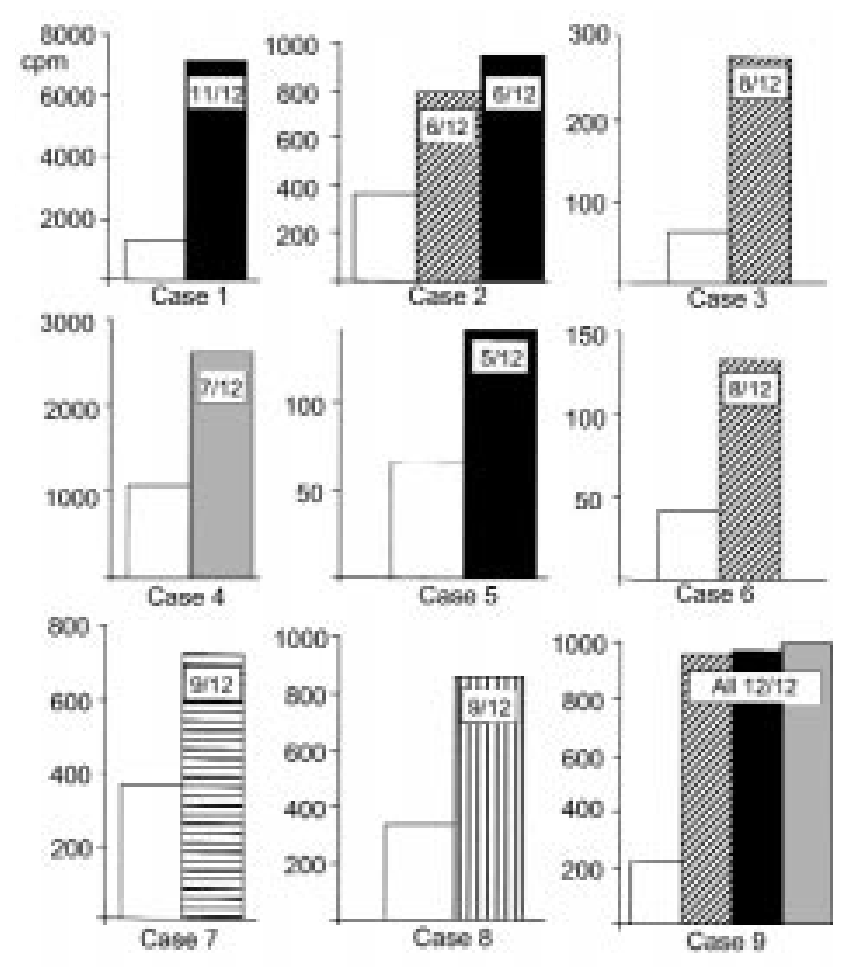

\section{Figure 5}

T-cell responses to IA-2ic synthetic peptides with sequences based on NPPEs identified by elution from HLA-DR4. Vertical axes show T-cell proliferation in cpm. Case numbers $1-9$ refer to the HLA-DR4 type 1 DM patients 1-9 described in Table 1 who had positive responses to IA-2ic peptides. Bars represent mean cpm from 12 replicate wells, and notations on bars indicate number of test wells positive (> mean +2 SD of background wells). Background, open bars; peptide 654-674, hatched bars; peptide 709-732, vertically striped bar; peptide 797-817, filled bars; peptide 854 872 , gray bars; and peptide $955-975$, horizontally striped bars. 
Table 2

Patients with type 1 DM and control subjects: HLA-DRB1 genotype and

T-cell responses to eluted IA-2 peptides



$\begin{array}{lllll}654-674 & 709-732 & 797-817 & 854-872 & 955-975\end{array}$

HLA DR4

Type 1 DM patients

\begin{tabular}{|c|c|c|c|c|c|c|c|c|}
\hline 1 & 17 & 8 & $0401 / 0101$ & + & & & POS $^{A}$ & \\
\hline 2 & 26 & 12 & $0401 / 1302$ & + & POS & & POS & \\
\hline 3 & 28 & 28 & $0401 / 1101$ & + & POS & & & \\
\hline 4 & 29 & 3 & $0401 / 0403$ & - & & & & POS \\
\hline 5 & 42 & 16 & $0401 / 0401$ & + & & & POS & \\
\hline 6 & 20 & 4 & $0401 / 0401$ & + & POS & & & \\
\hline 7 & 19 & 4 & $0401 / 0301$ & - & & & & \\
\hline 8 & 6 & 4 & $0403 / 0405$ & + & & POS & & \\
\hline 9 & 13 & $<1$ & $0403 / 0405$ & + & POS & & POS & POS \\
\hline 10 & 36 & 16 & $0401 / 0404$ & + & & & & \\
\hline 11 & 15 & 12 & $0401 / 0401$ & - & & & & \\
\hline 12 & 16 & 4 & $0401 / 0301$ & + & & & & \\
\hline 13 & 24 & 1 & $0401 / 1302$ & + & & & & \\
\hline \multicolumn{9}{|c|}{$\begin{array}{l}\text { Type } 1 \text { DM patients } \\
\text { (non-DR4) }\end{array}$} \\
\hline 14 & 28 & 4 & $0102 / 0301$ & - & POS & & & POS \\
\hline 15 & 20 & 25 & 0301 & - & & & & \\
\hline 16 & 16 & 12 & $1201 / 1301$ & + & & & & \\
\hline 17 & 10 & 12 & $0101 / 1101$ & - & & & & \\
\hline 18 & 13 & 2 & $0301 / 1301$ & + & & & & \\
\hline 19 & 23 & 1 & $1101 / 1301$ & - & & & & \\
\hline 20 & 24 & 25 & $0301 / 0901$ & - & & & & \\
\hline 21 & 33 & 20 & $0301 / 08$ & - & & & & \\
\hline \multicolumn{9}{|c|}{$\begin{array}{l}\text { HLA-DR4 } \\
\text { Controls }\end{array}$} \\
\hline 22 & 36 & - & 0401/0101 & - & & & & \\
\hline 23 & 17 & - & $0401 / 0101$ & - & & & & \\
\hline 24 & 30 & - & $0401 / 1302$ & - & & & & \\
\hline 25 & 24 & - & $0401 / 1501$ & - & & & & \\
\hline 26 & 40 & - & $0401 / 14$ & - & & & & \\
\hline 27 & 16 & - & $0401 / 0403$ & - & & & & \\
\hline 28 & 24 & - & $0401 / 02$ & - & & & & \\
\hline 29 & 30 & - & $0401 / 0701$ & - & & & & \\
\hline
\end{tabular}

APOS indicates a T-cell response to peptide (simulation index $>2.0$; significant number of wells positive $[P<0.05])$.

partial sequence information, to a combination of the ADS and highly sensitive MALDI-TOF MS analysis. The use of PWM does not appear to limit class II antigen processing, because the IA-2ic was observed to be processed into nested sets with extensions and truncations at the $\mathrm{NH}_{2}$ - and $\mathrm{COOH}$ - termini, consistent, we believe, with all MHC class II naturally processed peptide identification studies published to date using various murine and human APCs, both in vitro and in vivo $(26,40,46)$. However, the effects of PWM on antigen processing are not known, and the ADS we use may give rise to processing and presentation that is not entirely representative of the corresponding process in patient APCs, for example by restricting uptake of antigen to a particular pathway.

We describe the successful elution and identification of femtomole quantities of peptides that are presented after natural processing of a specific Ag through the HLA class II pathway, providing an HLA allele-specific map of IA-2ic NPPEs. Our approach identifies 6 HLADR4-restricted NPPE regions in IA-2ic, each of which elicits a T-cell response and 5 of which match peptides described by others as eliciting T-cell responses in HLADR4 (0401) homozygous first-degree relatives of type 1
DM patients $(28,34)$, whereas 1 (652$680)$ is described here for the first time. We are not aware of any additional HLA-DR4-restricted epitopes of IA-2ic described elsewhere. Taken in concert, these results suggest that our approach is capable of identifying immunologically relevant, disease-related NPPEs of a given Ag, presented by a given HLA class II molecule. Although we eluted IA-2 peptides of both low and high affinity for HLA-DR4, we cannot exclude the possibility that our approach is not sufficiently sensitive to detect minor or low-abundance T-cell epitopes. It is not surprising that T-cell proliferation was not detected in all HLA-DR4 type 1 DM patients because the precursor frequency of islet autoreactive $T$ cells capable of proliferating against a single epitope in peripheral blood is likely to be very low. Nonetheless, T-cell responses to IA-2ic NPPEs show a high degree of sensitivity and specificity for type $1 \mathrm{DM}$, in contrast with other reports measuring $\mathrm{T}$-cell responses to overlapping synthetic peptides of islet autoantigens $(47,48)$. Our results also stress the importance of HLA allele-specific epitope mapping for CD4 T cells, because only 1 of the non-HLA-DR4 type 1 DM patients showed a T-cell proliferative response to the DR4-specific NPPEs.

The technique described represents a powerful approach to epitope identification, with the major advantages of being highly flexible and widely applicable for identification of NPPEs presented by any selected class II MHC molecule processed from any Ag. Application of the approach to HLA-DQ8-presented NPPEs will be particularly useful in type $1 \mathrm{DM}$, which is strongly associated with HLA-DQ alleles; to date the cloning of HLA-DQ8-restricted autoreactive CD4 T cells in type $1 \mathrm{DM}$ has proved particularly difficult. Finally, the approach will be of particular importance in the future exploitation of epitopes recognized by CD4 T cells, which represent potential therapeutic agents, either in native form (49) or as antagonistic altered peptide ligands (8), for the treatment of autoimmune and inflammatory disease. Certain knowledge of NPPEs will also be important in the exploitation of Ag-specific CD4 T-cell responses in autoimmune disease prediction, as surrogate end-points in immunotherapeutic trials in autoimmune disease, and as part of the developing area of HLA tetramer technology (50).

\section{Acknowledgments}

This work was supported by the award of a Senior Clinical Fellowship to M. Peakman by the British Diabetic Association. We are grateful to Michael Christie for measurement of IA-2ic autoantibodies and the gift of 
the IA-2ic cDNA, and to Constantino Pitzalis and David Scott for help in recruiting patients.

1. Germain, R.N. 1994. MHC-dependent antigen processing and peptide presentation: providing ligands for $\mathrm{T}$ lymphocyte activation. Cell. 76:287-299.

2. Zerva, L., et al. 1996. Arginine at positions 13 or 70-71 in pocket 4 of HLA-DRB1 alleles is associated with susceptibility to tuberculoid leprosy. J. Exp. Med. 183:829-836.

3. Cramp, M.E., et al.. 1998. Association between HLA class II genotype and spontaneous clearance of hepatitis C viraemia. J. Hepatol. 29:207-213.

4. Pardoll, D.M., and Topalian, S.L. 1998. The role of CD4+ T cell responses in antitumor immunity. Curr. Opin. Immunol. 10:588-594.

5. Czarnecki, D., Nicholson, I., Tait, B., and Nash, C. 1993. HLA DR4 is associated with the development of multiple basal cell carcinomas and malignant melanoma. Dermatology. 187:16-18.

6. Hammer, J., Torniolo, T., and Sinigaglia, F. 1997. HLA class II peptide binding specificity and autoimmunity. Adv. Immunol. 66:67-100.

7. Wucherpfennig, K.W., and Strominger, J.L. 1995. Molecular mimicry in $\mathrm{T}$ cell-mediated autoimmunity: viral peptides activate human $\mathrm{T}$ cell clones specific for myelin basic protein. Cell. 80:695-705.

8. Collins, E.J., and Frelinger, J.A. 1998. Altered peptide ligand design: altering immune responses to class I MHC/peptide complexes. Immunol. Rev. 163:151-160.

9. Davenport, M.P., and Hill, A.V. 1996. Reverse immunogenetics: from HLA-disease associations to vaccine candidates. Mol. Med. Today. 2:38-45.

10. Watts, C. 1997. Capture and processing of exogenous antigens for presentation on MHC molecules. Annu. Rev. Immunol. 15:821-50.

11. Chicz, R.M., et al. 1992. Predominant naturally processed peptides bound to HLA-DR1 are derived from MHC-related molecules and are heterogeneous in size. Nature. 358:764-768.

12. Bartnes, K., Leon, F., Briand, J.P., Travers, P.J., and Hannestad, K. 1999. $\mathrm{N}$-terminal elongation of a peptide determinant beyond the first primary anchor improves binding to H-2 I-Ad and HLA-DR1 by backbonedependent and aromatic side chain-dependent interactions, respectively. Eur. J. Immunol. 29:189-195.

13. Carson, R.T., Desai, D.D., Vignali, K.M., and Vignali, D.A. 1999. Immunoregulation of Th cells by naturally processed peptide antagonists. J. Immunol. 162:1-4.

14. Carson, R.T., Vignali, K.M., Woodland, D.L., and Vignali, D.A. 1997. T cell receptor recognition of MHC class II-bound peptide flanking residues enhances immunogenicity and results in altered TCR $V$ region usage. Immunity. 7:387-399.

15. Castano, L., and Eisenbarth, G.S. 1990. Type-I diabetes: a chronic autoimmune disease of human, mouse, and rat. Annu. Rev. Immunol. 8:647-679.

16. She, J.X. 1996. Susceptibility to type I diabetes: HLA-DQ and DR revisited. Immunol. Today. 17:323-329.

17. Leslie, R.D., Atkinson, M.A., and Notkins, A.L. 1999. Autoantigens IA-2 and GAD in type I (insulin-dependent) diabetes. Diabetologia. 42:3-14.

18. Kawasaki, E., et al. 1997. Evaluation of islet cell antigen (ICA) 512/IA-2 autoantibody radioassays using overlapping ICA512/IA-2 constructs. J. Clin. Endocrinol. Metab. 82:375-380.

19. Christie, M.R., et al. 1994. Antibodies to islet $37 \mathrm{k}$ antigen, but not to glutamate decarboxylase, discriminate rapid progression to IDDM in endocrine autoimmunity. Diabetes. 43:1254-1259.

20. Faas, S.J., Menon, R., Braun, E.R., Rudert, W.A., and Trucco, M. 1996 Sequence-specific priming and exonuclease-released fluorescence detection of HLA-DQB1 alleles. Tissue Antigens. 48:97-112.

21. Payton, M., Hawkes, C.J., and Christie, M.R. 1995. Relationship of the 37,000 and 40,000-Mr tryptic fragments of islet antigens in insulindependent diabetes to the protein tyrosine phosphatase-like molecule IA-2 (ICA512). J. Clin. Invest. 96:1506-1511.

22. Stevens, E.J., and Peakman, M. 1998. Enhanced T cell proliferation and increased responder frequency following delivery of antigen to the antigen presenting cell; B cell dependency and use in detection of autoreactive T cells. J Immunol. Methods. 215:59-70.

23. Chilson, O.P., and Kelly-Chilson, A.E. 1989. Mitogenic lectins bind to the antigen receptors on human lymphocytes. Eur. J. Immunol. 19:389-396.

24. Peakman, M., et al. 1994. T cell clones generated from patients with Type 1 diabetes using interleukin-2 proliferate to human islet antigens. Autoimmunity. 17:31-39.

25. Gorga, J.C., Horejsi, V., Johnson, D.R, Raghupathy, R., and Strominger, J.L. 1987. Purification and characterization of class II histocompatibility antigens from a homozygous B cell line. J. Biol. Chem 262:16087-16094.
26. Chicz, R.M., et al. 1993. Specificity and promiscuity among naturally processed peptides bound to HLA-DR alleles. J. Exp. Med. 178:24-47.

27. Chicz, R.M., Graziano, D.F, Trucco, M., Strominger, J.L., and Gorga, J.C. 1997. HLA-DP2: self peptide sequences and binding properties. $J$. Immunol. 159:4935-4942.

28. Honeyman, M.C., Stone, N.L., and Harrison, L.C. 1998. T-cell epitopes in type 1 diabetes autoantigen tyrosine phosphatase IA-2: potential for mimicry with rotavirus and other environmental agents. Mol. Med. 4:231-239.

29. Lohmann, T., et al. 1999 . T cell reactivity to $\mathrm{DR}^{*} 0401$ and $\mathrm{DQ} * 0302$ binding peptides of the putative autoantigen IA-2 in type 1 diabetes. Exp. Clin. Endocrinol. Diabetes. 107:166-171.

30. Hammer, J., et al. 1994. Precise prediction of major histocompatibility complex class II peptide interactions based on side chain scanning. J. Exp. Med. 180:2353-2358.

31. Marsh, S.G. 1998. HLA class II region sequences. Tissue Antigens. 51:467-507.

32. Roep, B.O., et al. 1996. HLA-associated inverse correlation between T cell and antibody responsiveness to islet autoantigen in recent-onset insulindependent diabetes mellitus. Eur. J. Immunol. 26:128-1289.

33. Harrison, L.C., et al. 1993. Inverse relation between humoral and cellular immunity to glutamic acid decarboxylase in subjects at risk of insulin-dependent diabetes. Lancet. 341:136-1369.

34. Honeyman, M.C., Brusic, V., Stone, N.L., and Harrison, L.C. 1998. Neural network-based prediction of candidate T-cell epitopes. Nat. Biotechnol. 16:966-969.

35. Liu, G.Y., et al. 1995. Low avidity recognition of self-antigen by T cells permits escape from central tolerance. Immunity. 3:407-415.

36. Endl, J., et al. 1997. Identification of naturally processed T cell epitopes from glutamic acid decarboxylase presented in the context of HLA-DR alleles by $\mathrm{T}$ lymphocytes of recent onset IDDM patients. J. Clin. Invest. 99:240-2415.

37. Vincent, A., and Willcox, N. 1994. Characterization of specific T cells in myasthenia gravis. Immunol. Today. 15:41-42.

38. Nagvekar, N., et al. 1999. Scanning a DRB3*0101 (DR52a)-restricted epitope cross-presented by DR3: overlapping natural and artificial determinants in the human acetylcholine receptor. J. Immunol. 162:4079-4087.

39. Stevens, E.J., and Peakman, M. 1997. T-cell studies in insulin dependent diabetes mellitus: lessons from other autoimmune diseases. Diabetes. Nutr. Metab. 10:242-254.

40. Rudensky, A.Y., Preston-Hurlburt, P., Hong, S.C, Barlow, A., and Janeway, C.A. 1991. Sequence analysis of peptides bound to MHC class II molecules. Nature. 353:622-627.

41. Demotz, S., Grey, H.M., Appella, E., and Sette, A. 1989. Characterization of a naturally processed MHC class II-restricted T-cell determinant of hen egg lysozyme. Nature. 342:682-684.

42. Vignali, D.A., Urban, R.G., Chicz, R.M., and Strominger, J.L. 1993. Minute quantities of a single immunodominant foreign epitope are presented as large nested sets by major histocompatibility complex class II molecules. Eur. J. Immunol. 23:1602-1607.

43.Richter, W., Shi, Y., and Baekkeskov, S. 1993. Autoreactive epitopes defined by diabetes-associated human monoclonal antibodies are localized in the middle and C-terminal domains of the smaller form of glutamate decarboxylase. Proc. Natl. Acad. Sci. USA. 90:2832-2836.

44. Tremble, J., et al. 1997. Human B cells secreting immunoglobulin G to glutamic acid decarboxylase- 65 from a nondiabetic patient with multiple autoantibodies and Graves' disease: a comparison with those present in type 1 diabetes. J. Clin. Endocrinol. Metab. 82:2664-2670.

45. Phelps, R.G., Turner, A.N., and Rees, A.J. 1996. Direct identification of naturally processed autoantigen-derived peptides bound to HLADR15*. J. Biol. Chem. 271:18549-18553.

46. Marrack, P., Ignatowicz, L., Kappler, J.W., Boymelm, J., and Freed, J.H. 1993. Comparison of peptides bound to spleen and thymus class II. J. Exp. Med. 178:2173-2183.

47. Lohmann, T., et al. 1994. Immunodominant epitopes of glutamic acid decarboxylase 65 and 67 in insulin-dependent diabetes mellitus. Lancet. 43:1607-1608.

48. Atkinson, M.A., et al. 1994. Cellular immunity to a determinant common to glutamate decarboxylase and coxsackie virus in insulin-dependent diabetes. J. Clin. Invest. 94:2125-2129.

49. Xu, X.J., et al. 1999. Spontaneous T cell proliferation in the non-obese diabetic mouse to a peptide from the unique class II MHC molecule, I$\mathrm{A}^{\mathrm{g}}$, which is also protective against the development of autoimmune diabetes. Diabetologia. 42:560-565

50. Crawford, F., Kozono, H., White, J., Marrack, P., and Kappler, J. 1998. Detection of antigen-specific $\mathrm{T}$ cells with multivalent soluble class II MHC covalent peptide complexes. Immunity. 8:675-682. 